\title{
Crosslinked Polyethyleneimine Gel Polymer Interface to Improve Cycling Stability of RFBs
}

\author{
Hyung-Seok Lim $\mathbb{D}^{1},{ }^{1}$ Sujong Chae, ${ }^{1}$ Litao Yan, ${ }^{1}$ Guosheng Li $\left(\mathbb{D},{ }^{1}\right.$ Ruozhu Feng $\left(\mathbb{D},{ }^{1}\right.$ \\ Yongsoon Shin, ${ }^{2}$ Zimin Nie, ${ }^{1}$ Bhuvaneswari Modachur Sivakumar, ${ }^{1}$ Xin Zhang ${ }^{\mathbb{D}},{ }^{2}$ \\ Yangang Liang, ${ }^{1}$ David Jonathan Bazak $\mathbb{D}^{2},{ }^{2}$ Vaithiyalingam Shutthanandan $\mathbb{D}^{2}$, \\ Vijayakumar Murugesan $\mathbb{D}^{2},{ }^{2}$ Soowhan Kim, ${ }^{1}$ and Wei Wang $\mathbb{D}^{1}$ \\ ${ }^{1}$ Energy and Environment Directorate, Pacific Northwest National Laboratory, Richland, WA 99354, USA \\ ${ }^{2}$ Physical \& Computational Science Directorate, Pacific Northwest National Laboratory, Richland, WA 99354, USA
}

Correspondence should be addressed to Wei Wang; wei.wang@pnnl.gov

Received 11 May 2021; Accepted 12 October 2021; Published 5 January 2022

Copyright (C) 2022 Hyung-Seok Lim et al. Exclusive Licensee Beijing Institute of Technology Press. Distributed under a Creative Commons Attribution License (CC BY 4.0).

\begin{abstract}
Redox flow batteries are considered a promising technology for grid energy storage. However, capacity decay caused by crossover of active materials is a universal challenge for many flow battery systems, which are based on various chemistries. In this paper, using the vanadium redox flow battery as an example, we demonstrate a new gel polymer interface (GPI) consisting of crosslinked polyethyleneimine with a large amount of amino and carboxylic acid groups introduced between the positive electrode and the membrane. The GPI functions as a key component to prevent vanadium ions from crossing the membrane, thus supporting stable long-term cycling. Cyclic voltammetry (CV) and electrochemical impedance spectroscopy (EIS) measurements were conducted to investigate the effect of GPI on the electrochemical properties of graphitic carbon electrodes (GCFs) and redox reaction of catholyte. X-ray photoelectron spectroscopy (XPS) and ${ }^{1} \mathrm{H}$ nuclear magnetic resonance (NMR) spectra demonstrated that the crosslinked GPI is chemically stable for 100 cycles without dissolution of polymers and swelling in the strong acidic electrolytes. Results from inductively coupled plasma mass spectrometry (ICP-MS), Fourier-transform infrared (FTIR) spectroscopy, and energy-dispersive X-ray (EDX) spectroscopy proved that the GPI is effective in maintaining the concentration of vanadium species in their respective half-cells, resulting in improved cycling stability because of it prevents active species from crossing the membrane and stabilizes the oxidation states of active species.
\end{abstract}

\section{Introduction}

Because of their ability to store and release large amounts of energy in a controlled manner, stationary energy storage systems have become increasingly important for renewable energy integration and grid modernization. Among various technologies, redox flow batteries (RFBs) have received much attention as one of the most promising candidates for stationary energy storage because of their inherent safety, decoupled power/energy, and long service life [1-3]. However, a universal challenge for RFB technologies is the substantial capacity fading caused by crossover of active species from one halfcell to the other through the membrane, especially over long-term operations [4-6]. Constant capacity decay requires the system to be overdesigned to deliver the rated power/ energy, as well as implementation of extra system and operational measures to ensure stable cycling.

Material degradation and side reactions obviously can lead to capacity decay [7]. Another major contribution however is crossover of active materials $[2,8,9]$. For example, one of the most highly developed RFB technologies is the vanadium redox flow battery (VRB) [6], which traditionally uses a proton-conductive membrane such as Nafion $^{\mathrm{TM}}$ to separate the two half-cells. More detailed mechanistic study suggests that the main reasons for capacity decay in a VRB during cycling are imbalanced crossover of vanadium active species and asymmetrical valence of vanadium ions in the electrolytes caused by self-discharge reactions after crossover [10]. Many research efforts have been devoted to the development of new membranes or modification of Nafion ${ }^{\mathrm{TM}}$ to 


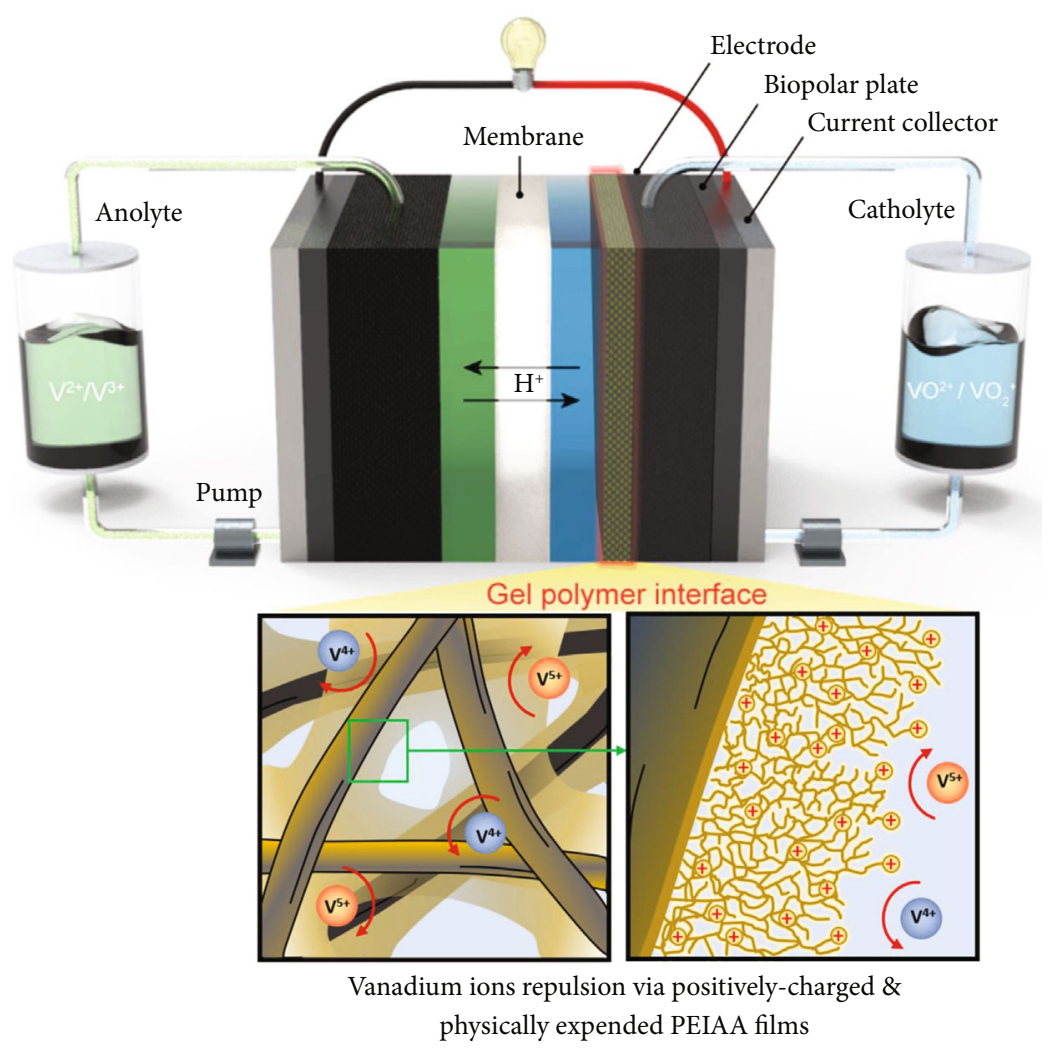

Scheme 1: Schematic illustration of the multifunctional effects of a PEIAA GPI introduced on the GCF to prevent crossover of vanadium ions in the VRB.

achieve better selectivity [11-14] (i.e., reducing crossover). For example, amine polymer-based membranes have been studied for VRBs because of their surface chemistry with positively charged groups to repel vanadium ions via Donnan exclusion in strong acid conditions [15-18]. Even though some of those membranes improved cycling performance by reducing permeability rates of vanadium ions, their complicated fabrication or modification processes may result in high cost. The cationic-charged polymer films or surface coating on a membrane acting as an electrical repulsion barrier also disturbs proton permeation, thus leading to higher area resistance [19]. Most current efforts to overcome the capacity decay issue are almost exclusively focused on membrane development and modification [20-22]. New approaches are rarely reported and discussed. Nevertheless, capacity decay remains a key challenge.

In its role of separating the positive and negative electrolytes, a membrane must provide both high conductivity towards charge transfer ions and high selectivity towards energy-bearing active species. These two competing requirements mean the structure and morphology of the membrane must be designed to achieve two opposing functionalities. Improvement of one function often is achieved at the sacrifice of the other. In light of this inherent challenge, we postulate a new component that can be introduced into the traditional RFB structure to prevent crossover of active species, thus providing more latitude for material selection and system design for both the membrane and the flow cell.
Using a VRB as an example, we created a gel polymer interface (GPI) consisting of crosslinked polyethyleneimine (PEI) with amino and the oxygen functional groups such as carboxylic acid and carbonyl and hydroxyl groups between the electrode and membrane to effectively prevent electrolyte crossover. We found that the as-synthesized GPI also increases the surface wettability of a carbon felt electrode because of its hydrophilicity. The PEI with amino and carboxylic acids (PEIAAs) in acidic conditions acts to not only repulse vanadium ion with expended polymer networks but to consequently stabilize the oxidation state of vanadium ions with positively charged amines. The PEIAA GPI with abundant amounts of primary, secondary, and tertiary amines and also oxygen-containing groups that are mainly carboxylic acid groups preserves almost the same concentration of negative and positive electrolytes even after 100 cycles and resulted in five times higher cycling stability in capacity retention $\left(86.46 \%\right.$ at the $100^{\text {th }}$ cycle, a capacity fading rate $0.16 \%$ /cycle) with higher Coulombic efficiency (CE) $\left(98.37 \%\right.$ at the $100^{\text {th }}$ cycle) than a pristine graphitic carbon felt (GCF) cell $\left(17.65 \%\right.$ at the $100^{\text {th }}$ cycle with a CE of $96.73 \%$, capacity fading rate of $0.81 \% /$ cycle). A schematic illustration of a novel GPI system in a VRB is presented in Scheme 1.

\section{Results and Discussion}

The chemical reaction that yields the PEIAA is shown in Figure 1 . 

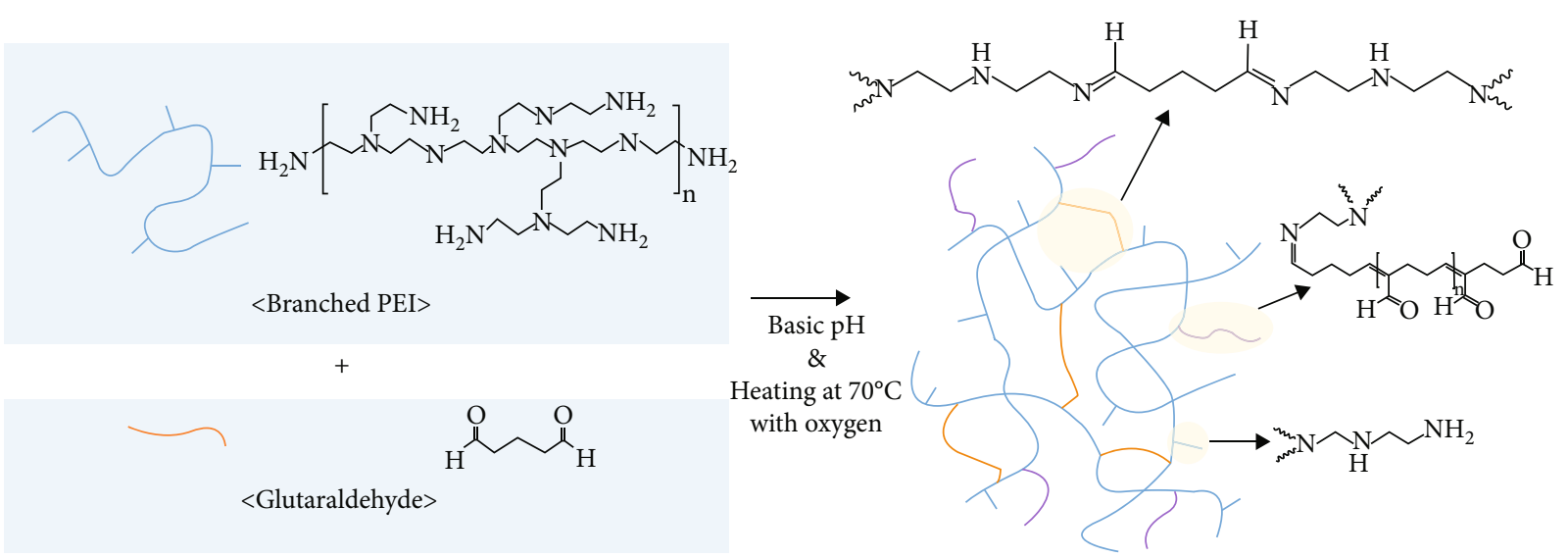

FIGURE 1: Chemical structures of PEI and GA and their chemical reaction to form crosslinked regions and amino and carboxylic acid groups.

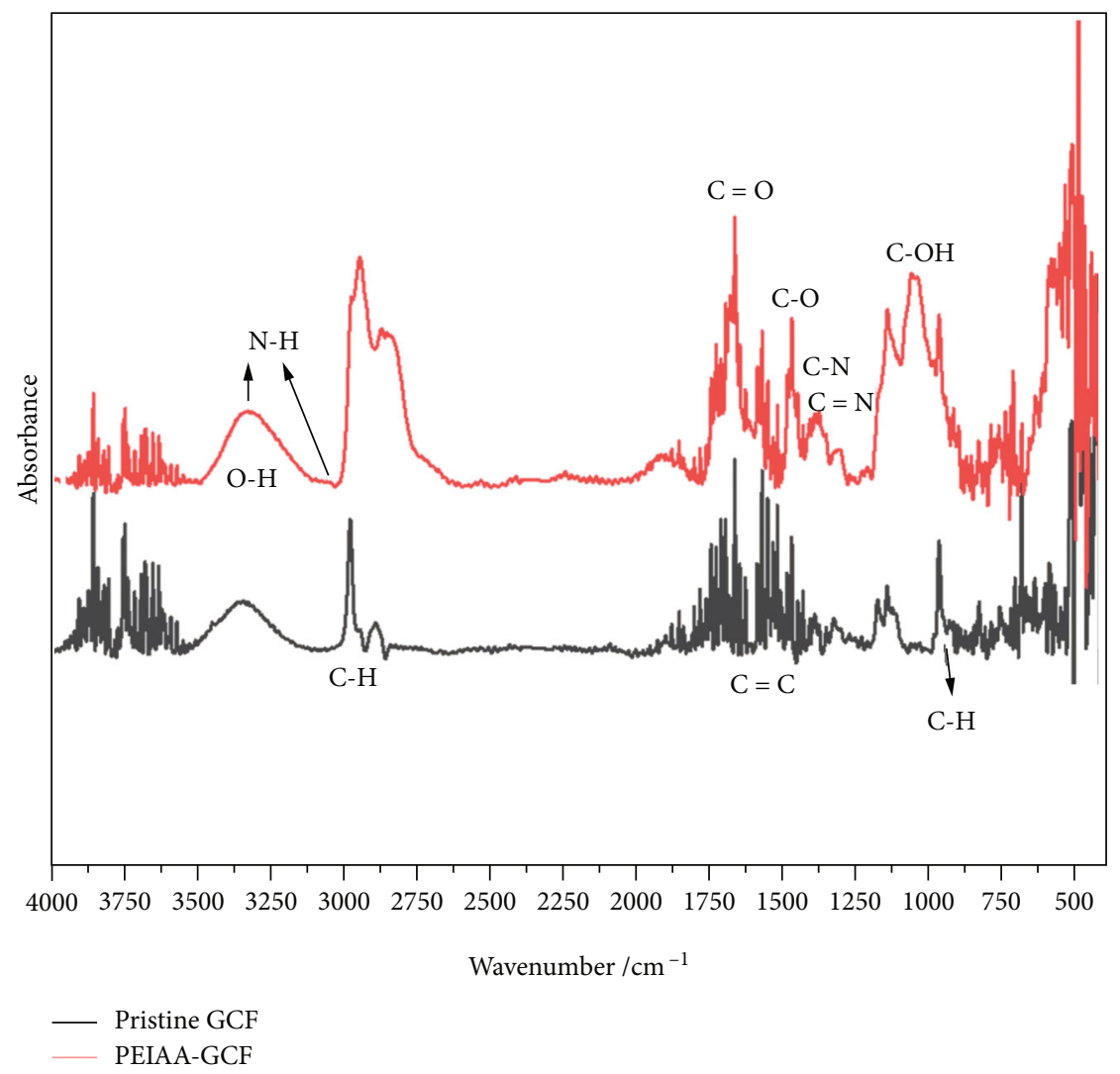

FIGURE 2: FTIR spectra of (black) pristine GCF and (red) PEIAA-GCF samples.

GA played two roles in this study. Uncrosslinked (linear) PEI is not stable in aqueous solutions because thermodynamically compatible water molecules easily diffuse into the PEI phase and disentangle them (see Fig. S1). Thus, GA was used as a crosslinker to stabilize the PEI under strong acidic conditions [23] because it readily reacts with amine-containing molecules such as PEI through the formation of Schiff bases. Thus, a GPI with a swellable three dimensionally crosslinked structure is obtained. GA molecules in aqueous solutions also form aldehyde polymers. In the drying step for the GCF samples treated in PEI and
GA solutions at $70^{\circ} \mathrm{C}$ in air, aldehydes are readily oxidized via the following reaction to form carboxylic acid groups:

$$
2 \mathrm{RCHO}+\mathrm{O}_{2} \longrightarrow 2 \mathrm{RCOOH}
$$

FTIR spectra of pristine GCF and PEIAA-GCF samples reveal that the PEIAA-GCF sample had amino and carboxylic acid groups (amines and carboxylic acids). In the case of the pristine GCF, Figure 2 shows oxygen-containing groups in the PEIAA-GCF over a very broad band in the $3300-2500 \mathrm{~cm}^{-1}$ region for hydroxyl $(\mathrm{O}-\mathrm{H})$ stretching, 

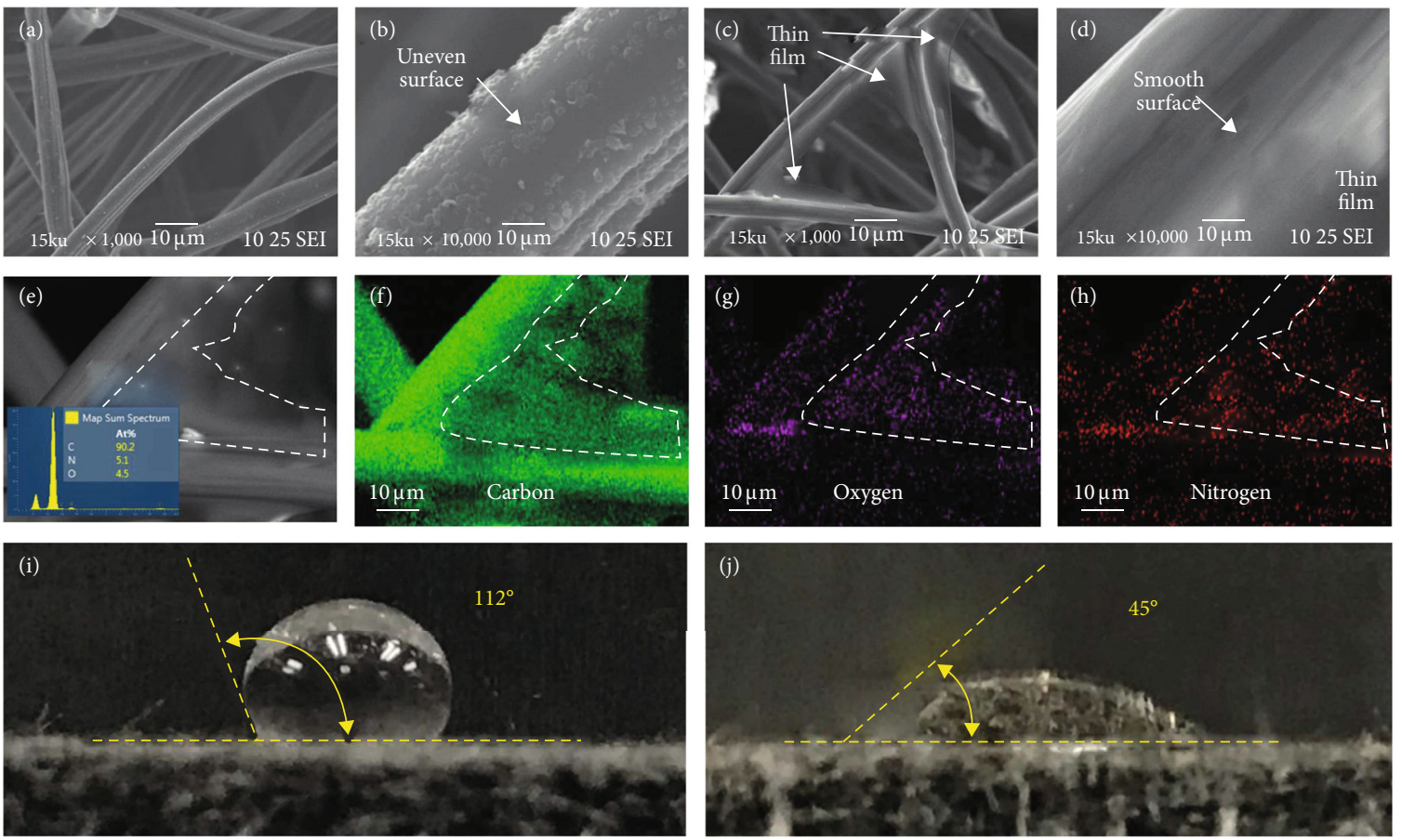

FIGURe 3: SEM images of (a, b) pristine GCF and (c, d) PEIAA-GCF samples with different magnifications (1,000x and 10,000x). SEM-EDX (e) spectrum and element maps ((f) carbon, (g) oxygen, and (h) nitrogen) of a part of PEIAA-GCF sample. Contact angles of (i) the pristine GCF and (j) the PEIAA-GCF samples.

intense bands from 1760 to $1690 \mathrm{~cm}^{-1}$ and $1320-1200 \mathrm{~cm}^{-1}$ corresponding to $\mathrm{C}=\mathrm{O}$ (polymeric aldehyde segment with carboxylic acids) [24, 25]. C-OH vibration comprised of a mixture of $\mathrm{C}-\mathrm{O}$ stretch and $\mathrm{C}-\mathrm{O}-\mathrm{H}$ bend with the appearance of typical N-H and C-N stretching vibrations corresponding to amine groups and PEI backbone. Therefore, the amino and carboxylic acid groups were successfully introduced into PEI backbones.

Figure 3 shows scanning electron microscopy (SEM) images of ( $a$ and $b$ ) pristine GCF and ( $c$ and $d$ ) PEIAAGCF electrodes under different magnifications.

After PEIAA modification using the immersion method and followed by oxidation in air, the string surface of the GCF became smooth with very thin polymer films around the carbon strings. Rapid reaction of aldehydes and primary amines of PEI dissolved in aqueous solutions by formation of Schiff bases makes the polymers partially hydrophobic so they have an affinity for the hydrophobic surface of the carbon strings [23]. After the formation of crosslinked PEI with GA, we dried the samples in air at $70^{\circ} \mathrm{C}$ for 12 hours in an oven to oxidize the aldehyde groups to carboxylic acid groups on the polymer backbone. The SEM images show that the uneven surface of the pristine GCF fibers changed to a smooth surface with a spider web-like polymer phase, thus demonstrating our approach to be a simple and effective way to coat graphite fibers in the widely used GCF electrode. Figures $3(\mathrm{e})-3(\mathrm{~h})$ show a SEM-EDX spectrum (in Figure 3(e)) and element maps of the PEIAA-GCF sample. The polymer phase in the PEIAA-GCF sample consists of 90.2\% (atomic) carbon, 4.5\% oxygen existing in aldehyde polymer regions and carboxylic acids, and $5.1 \%$ of nitrogen in the PEI regions, while pristine GCF is mostly carbon itself as shown in Fig. S2. Basically, the crosslinked PEI with GA does not have oxygen because the oxygens of aldehydes are removed by formation of Schiff bases with primary amines of PEI [21]. In this study, however, because GA caused aldehyde polymers to form with large amount of aldehyde groups, and after samples were dried at $70^{\circ} \mathrm{C}$ in air, aldehydes were oxidized to carboxylic acids to improve the surface hydrophilic property for better wettability of the GCF electrode, as explained above [26]. Therefore, the oxygen in the aldehyde and carboxylic acid groups was detected by EDX and FTIR. Figures 3(i) and 3(j) show the contact angles with water drops for the comparison of surface characteristics of GCF electrodes before and after PEIAA modification. The contact angle of pristine GCF was significantly reduced from $112^{\circ}$ to $45^{\circ}$ after PEIAA modification because of the large amount of oxygen-contained functional groups that improve the GCF surface. The polymer content introduced in PEIAA-GCF is $\sim 1.8 \mathrm{wt} \%$, as measured by thermogravimetric analysis and presented in Fig. S3. The weight loss of PEIAA happens below $400^{\circ} \mathrm{C}$, which is similar to neat PEI and indicates that the crosslinking density of the PEIAA phase is not too high because of the formation of aldehyde polymer regions [27]. We performed Brunauer-EmmettTeller and Barrett-Joyner-Halenda analyses for pristine GCF and PEIAA-GCF samples to identify the graphite fiber surface physical property change induced by polymer modification. Fig. S4 and Table S1 demonstrate that the surface area and average pore size of the GCF became smaller and the pore volume also decreased after PEIAA modification. These results are consistent with the surface changes of the 


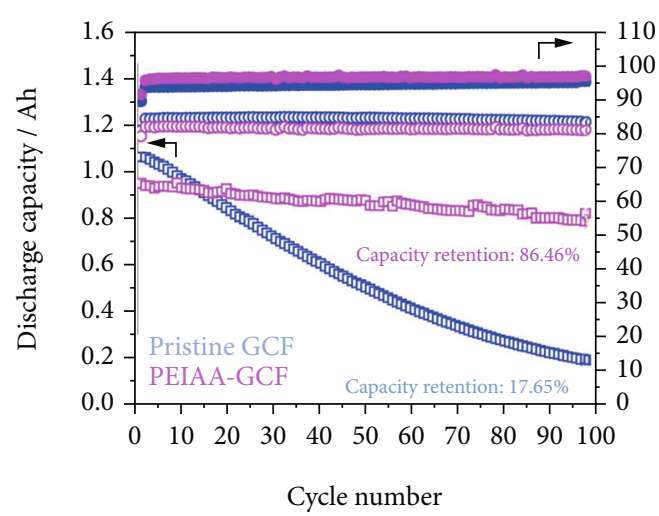

(a)

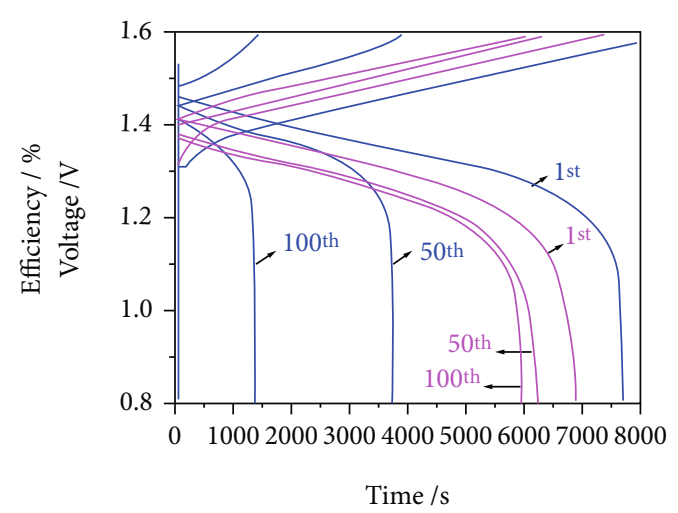

(b)

FIgure 4: (a, empty square) Discharge capacity, (a, solid circle) Coulombic efficiency, and (a, empty circle) energy efficiency; (b) voltagetime profiles of $1^{\text {st }}, 50^{\text {th }}$, and $100^{\text {th }}$ cycles of cells with (blue) pristine GCF and (pink) PEIAA-GCF electrodes under constant current charge at $50 \mathrm{~mA} \mathrm{~cm}^{-2}$.

samples before and after PEIAA modification as shown in the SEM images discussed above.

We observed the swelling response of PEIAA by immersing the sample in $0.9 \mathrm{M} \mathrm{H}_{2} \mathrm{SO}_{4}$ solution for 5 hours. Fig. S5 shows that large volumetric swelling of the PEIAA sample occurs in the strong acidic environment. The swelling was caused by the considerable number of water molecules that were absorbed in PEIAA phase. To verify the electrostatic repulsion of PEIAA, colloidal PEIAA solutions were prepared with PEIAA particles (Fig. S6a) in DI water and $0.9 \mathrm{M} \mathrm{H}_{2} \mathrm{SO}_{4}$ solution, respectively, and then, zeta potential was measured. The digital photographs of PEIAA colloidal solutions shown in Fig. S6b clearly show that the strongly acidic condition leads to particle swelling, which leads to murky solutions as indicated by the large proportion of the light scattered by swollen particle phase while the PEIAA solution with DI water is relatively clear. Fig. S6c shows that zeta potential of the PEIAA solution with $\mathrm{H}_{2} \mathrm{SO}_{4}$ became much higher $(47.2 \pm 5.4 \mathrm{mV})$ than the one with just DI water $(5.2 \pm 0.22 \mathrm{mV})$. This result suggests that PEIAA particles are more stable in acidic conditions because of the strong electrostatic interactions of particle surfaces that lead to a distribution of charged polymer branches in the system.

We tested the electrochemical performances of PEIAAGCF and pristine GCF electrodes in a flow cell with a traditional sulfuric vanadium electrolyte $\left(1.5 \mathrm{M} \mathrm{VOSO}_{4}\right.$ in $3.5 \mathrm{M}$ $\mathrm{H}_{2} \mathrm{SO}_{4}$ ). Testing was conducted within the voltage range of $0.8 \mathrm{~V}-1.6 \mathrm{~V}$ at a constant current density of $50 \mathrm{~mA} \mathrm{~cm}^{-2}$. Results from flow battery cycling tests of two flow cells with a pristine GCF and a PEIAA-GCF electrode are presented in Figure 4 . We conducted cycling tests to evaluate the effect of PEIAA modification on the cycling performance. Compared with the baseline GCF cell, the PEIAA-GCF cell exhibits better cell performance with much higher CE $(\sim 2 \%$ higher throughout 100 cycles) and most notably the significantly improved capacity retention $\left(86.46 \%\right.$ vs. $17.65 \%$ at $100^{\text {th }}$ cycle) shown in Figures 4(a) and 4(b). To investigate the electrochemical properties of the different GCF electrodes, the cyclic voltammetry (CV) curves were analyzed and displayed in Fig. S7. Both two different electrodes exhibit an anodic peak representing the oxidation of $\mathrm{VO}^{2+}$ to $\mathrm{VO}_{2}^{+}$ and a cathodic peak representing the reduction of $\mathrm{VO}^{2+}$ to $\mathrm{VO}_{2}{ }^{+}$. The current density of the PEIAA-GCF in the CV test is close to that of pristine GCF at both two different scan rates, which means the PEIAA modification does not interrupt the electron transferring. In addition, the smaller peak-to-peak separation of PEIAA-GCF electrode means that PEIAA modification gives rise to better chemical and electrochemical reversibility due to its better wettability leading to continuous stable reduction and reoxidation of analyte. Thus, PEIAA-GCF has no issue of electrochemical reversibility with a low barrier to electron transfer.

The energy efficiency of the flow cell with the PEIAAGCF is $\sim 2 \%$ lower than that of the baseline cell, possibly due to an increased internal resistance of the cell from reduced electrode conductivity, which is a result from the PEIAA on the GCF coating with its smaller pore volume and surface area. The effect of electrode modified with PEIAA interface on the positive $\mathrm{VO}^{2+} / \mathrm{VO}_{2}{ }^{+}$reaction was investigated by EIS measurement, as seen in Fig. S8. For the fresh cells, one more semicircle $\left(R_{\mathrm{sf}}\right.$, surface film resistance) appears for PEIAA-GCF cell with a larger charge transfer resistance $\left(R_{\mathrm{ct}}\right)$ while the pristine GCF shows only one semicircle due to the new interface in the PEIAA-GCF cell from the electrode modification. At low frequency, PEIAA-GCF fresh cell results in a decrease in mass transfer resistance $(W)$ due to the oxygen containing groups on PEIAA phase. The Nyquist plot of PEIAA-GCF cell at a SOC of 50\% shows the lower charge transfer resistance due to the better wettability. However, the smaller surface area and the decreased pore volume resulting from smaller pore size and reduced electrical conductivity as mentioned earlier might cause a larger charge transfer resistance at a SOC of $100 \%$ and consequently, the lower charge-discharge capacities with a larger cell overpotential at the initial cycle. Figure 4(b) shows voltage-time profiles of the GCF baseline and PEIAA-GCF cells at the $1^{\text {st }}, 50^{\text {th }}$, and $100^{\text {th }}$ cycles. The capacity decrease of pristine GCF cell is observed with increasing overpotential and decreasing charge-discharge time as cycling proceeds while cycling ability of the 
PEIAA-GCF cell is much more stable as seen in Figure 4(b). The poor cycling stability of the baseline cell could be attributed to both active species crossover and the subsequent increase of concentration polarization as cycling proceeds as previously reported [28]. In addition, the cycle performance tests prove that PEIAA is much more stable in vanadium electrolytes over 100 cycles.

To understand the mechanism of the GCF electrode modified with PEIAA on mitigation of the crossoverinduced capacity decay, we performed ICP-MS analysis of the cycled electrolytes. The results revealed that the pristine GCF cell has an asymmetric valence of redox-active species in the anolyte $\left(\mathrm{V}^{3+}\right)$ and decreasing concentration of $\mathrm{VO}^{2+}$ $\left(0.3 \mathrm{M} / \mathrm{L}, \sim 22 \%\right.$ remained at the $100^{\text {th }}$ cycle after discharge) in the catholyte during cycling. The imbalanced accumulation of $\mathrm{VO}_{2}{ }^{+}$in the positive side contributes to capacity fading. As a result, the amount of redox-active species $\mathrm{VO}^{2+}$ $(\mathrm{V}(\mathrm{IV}))$ in the positive side was significantly reduced and replaced with a high concentration of $\mathrm{VO}_{2}{ }^{+}(\mathrm{V}(\mathrm{V}))$ indicating the reaction became irreversible because of crossover of vanadium ions by possible diffusion, migration, electroosmotic convection, and subsequent side reactions in both electrolytes. Some of the potential reactions are listed in the below equations (2)-(5) [15]. Diffusion of vanadium ions through the membrane happens in the same direction regardless of charging and discharging. On the other hand, the direction of migration and electroosmotic convection is affected by charging or discharging. Different diffusion rates of vanadium ions can cause capacity fading due to a disparity between the state of charge of the anolyte and catholyte. Consequently, there is an accumulation of $\mathrm{VO}_{2}{ }^{+}$in the catholyte as cycling proceeds. In comparison, the PEIAA-GCF cell retains almost the same concentration of $\mathrm{VO}^{2+}$ $\left(1.45 \mathrm{M} / \mathrm{L} ; 299.4 \%\right.$ remained at the $100^{\text {th }}$ cycle) in the catholyte, leading to symmetric valence of redox-active species with a negligible amount of $\mathrm{VO}_{2}{ }^{+}$even after the $100^{\text {th }}$ discharge compared to the pristine cell. Because large amounts of amino groups in an abundance of PEI branches are protonated in strongly acidic conditions, strong positive charges prevent vanadium ion permeation by electrocharge repulsion. Besides, the significant expansion of PEIAA films with carboxylic acids helps limit undesired transport of vanadium ions and enhance electrochemical performance. To verify the effect of the PEIAA interface on reducing vanadium crossover, the $3.5 \mathrm{M} \mathrm{H}_{2} \mathrm{SO}_{4}$ solution was coupled with the catholyte $\left(1.5 \mathrm{M} \mathrm{V}^{4+}\right.$ in $3.5 \mathrm{M} \mathrm{H}_{2} \mathrm{SO}_{4}$ solution) and pumped through the cell where they are separated by Nafion 115 with pristine GCF or PEIAA-GCF electrodes for 5 days. UV-vis spectra were recorded for the concentrations of $\mathrm{V}^{4+}$ ions in $\mathrm{H}_{2} \mathrm{SO}_{4}$ solutions. The absorbance spectra at $765 \mathrm{~nm}$ of two samples revealed that the PEIAA interface led to $\sim 25 \%$ lower $\mathrm{V}^{4+}$ crossover, as displayed in Fig. S9b. Under no externally applied electric field, the results confirmed that the PEIAA interface on GCF slows crossover of $\mathrm{VO}_{2}{ }^{2+}$ ions via diffusion and convection. Along with the PEIAA-GCF electrode's ability to delay crossover, good wettability with aqueous electrolytes containing hydrated cationic vanadium ions with water molecules also is helpful to maintain good cycling stability. During charge and discharge processes, it is possi- ble that vanadium ions coordinate with water molecules, with one oxygen atom coming from sulfate and one carboxyl oxygen atom coming from amino and carboxylic acid in the PEIAA as previous studies suggest $[29,30]$.

Therefore, hyperbranched PEIAA networks with carboxylic acids present in GPI between the surfaces of the positive electrode and the Nafion membrane are effective in preventing vanadium ion crossover through the mechanisms of electrical repulsion from the protonated amines in acidic condition, physical hindrance from swollen PEIAA films in strong acidic electrolytes to prevent undesired vanadium crossover, and improved wettability with possible coordination chemistry of the amino and carboxylic acid functionality of PEIAA to stabilize the oxidation state of $\mathrm{VO}^{2+}$ and $\mathrm{VO}_{2}{ }^{+}$species.

$$
\begin{gathered}
\text { Negative side : } \mathrm{VO}^{2+}+\mathrm{V}^{2+}+2 \mathrm{H}^{+} \longrightarrow 2 \mathrm{~V}^{3+}+\mathrm{H}_{2} \mathrm{O} \\
\mathrm{VO}_{2}^{+}+2 \mathrm{~V}^{2+}+4 \mathrm{H}^{+} \longrightarrow 3 \mathrm{~V}^{3+}+2 \mathrm{H}_{2} \mathrm{O} \\
\text { Positive side }: \mathrm{V}^{3+}+\mathrm{VO}_{2}^{+} \longrightarrow 2 \mathrm{VO}^{2+} \\
\mathrm{V}^{2+}+\mathrm{VO}^{2+}+2 \mathrm{H}^{+} \longrightarrow 2 \mathrm{~V}^{3+}+\mathrm{H}_{2} \mathrm{O}
\end{gathered}
$$

We further analyzed the cycled electrodes and electrolytes using SEM, EDX, XPS, and ${ }^{1} \mathrm{H}-\mathrm{NMR}$. Fig. S10 and S11 show the smooth surface of carbon strings and the surviving polymer films detected with a EDX-nitrogen map after 100 cycles in vanadium electrolyte. Based on widescan XPS spectra, it is notable that $\mathrm{N} 1 \mathrm{~s}$ spectra appear only in PEIAA-GCF samples due to the tertiary amino groups of PEI while pristine GCF has no $\mathrm{N} 1 \mathrm{~s}$ signal, as shown in Figure 5 and Fig. S12.

XPS spectra of the PEIAA-GCF samples before and after 100 cycles also provide evidence that the crosslinked PEIAA is chemically stable in the strong acidic solution and oxidative environment of the $\mathrm{VO}_{2}{ }^{+}$ions in the charged catholyte. The nitrogen binding energies for the primary, secondary and tertiary amine peaks detected between $399 \mathrm{eV}$ and $401.7 \mathrm{eV}$ [31] are retained after 100 cycles, as shown in Figures 5(a) and 5(b). Since PEI chains are crosslinked with $\mathrm{GA}$ and the aldehyde groups are oxidized to carboxylic acid groups, $\mathrm{C}=\mathrm{N}$ and $\mathrm{COOH}$ bondings are also detected at $285.7 \mathrm{eV}$ and $288.5 \mathrm{eV}$, respectively. In the case of the pristine GCF sample before the cell test, there also are several peaks of $\mathrm{C} 1 \mathrm{~s}$ binding energies corresponding to $\mathrm{C}-\mathrm{OH}, \mathrm{C}$ $\mathrm{O}-\mathrm{C}, \mathrm{C}=\mathrm{O}$, and $\mathrm{COOH}$ frequencies at $286.11,286.72$, 287.32, and 288.14, respectively. However, the intensities of these peaks were reduced significantly after 100 cycles, as shown in Figure 5(c). Thus, organics containing oxygen atoms on the GCF surface were removed or decomposed in the strong acidic electrolytes during long-term cycling. This result may correspond to the surface change from rough to relatively smooth after cycling, as shown in Fig. S13. In contrast, all C $1 \mathrm{~s}$ binding energy peaks for PEIAAGCF that underwent the same testing conditions, as shown in Figure 5(d), also show that no polymer (PEIAA) dissolution occurs in the catholytes over 100 cycles, as shown in Fig. S14. Thus, the chemical robustness of GPI consisting of the 


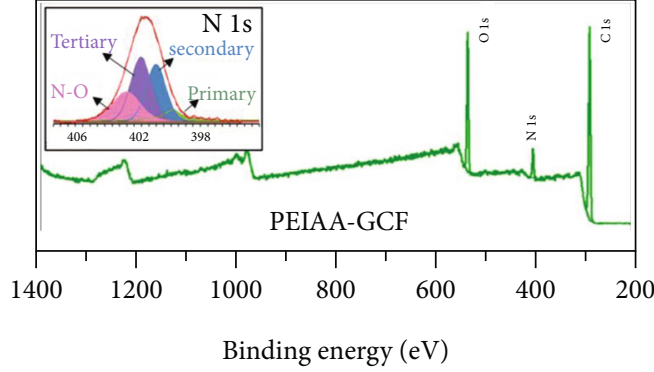

(a)
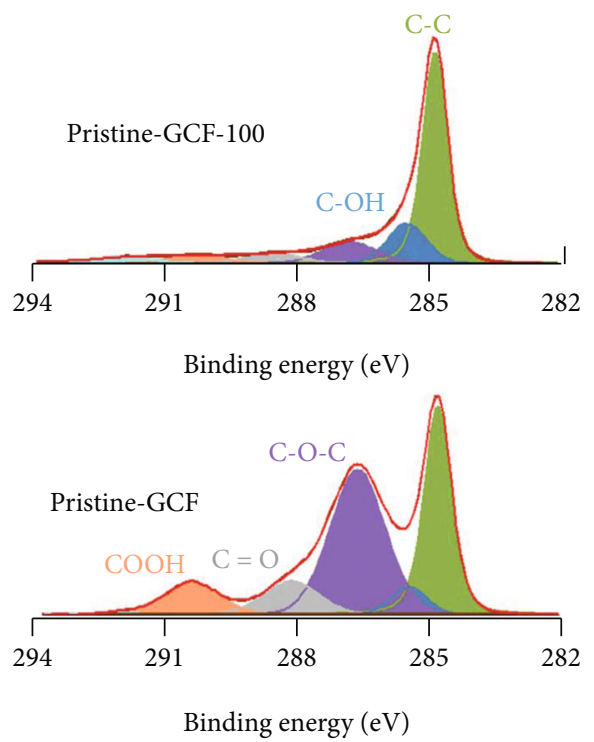

(c)

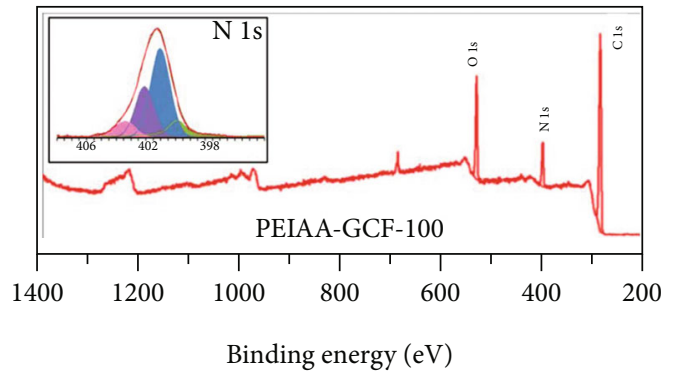

(b)
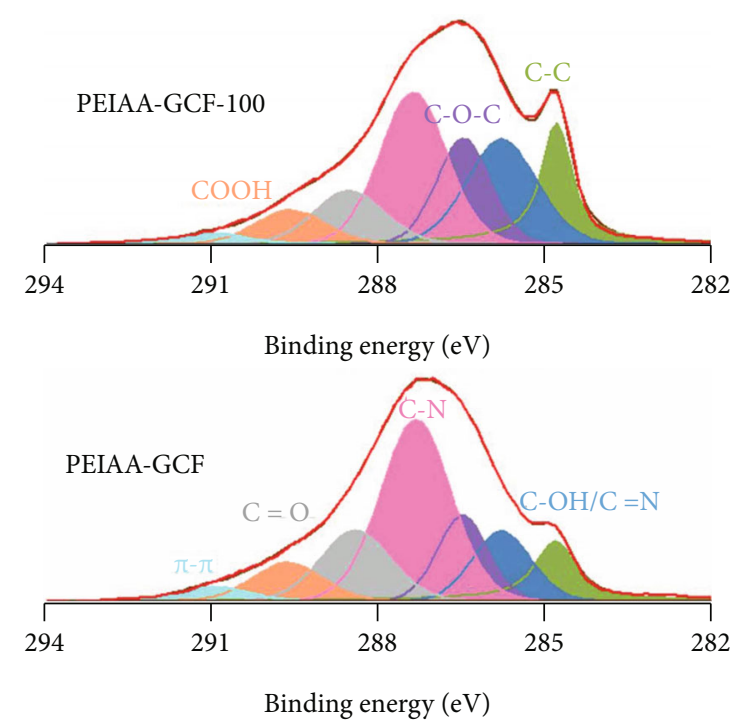

(d)

FIGURE 5: Wide scan XPS and inserted N 1 s spectra of PEIAA-GCF electrodes (a) before and (b) after 100 cycles under constant current charge at $50 \mathrm{~mA} \mathrm{~cm}^{-2}$. High-resolution XPS C $1 \mathrm{~s}$ spectra of (c) pristine GCF and (d) PEIAA-GCF electrodes before and after $100 \mathrm{cycles.}$

crosslinked PEIAA with carboxylic acids contributes to the long-term stability of vanadium redox flow battery cells.

\section{Conclusions}

We used a simple modification method to introduce a GPI to a chemically stable crosslinked PEIAA to the surface of a GCF acting as the positive electrode in a VRB. The PEIAA-GPI electrode demonstrated a significant blocking effect in preventing active vanadium species from crossover, thus providing more stable cycling performance. In contrast, serious imbalanced crossover and asymmetrical valence of active-species vanadium ions were observed in a cell using pristine GCF electrodes. The positive effect of the PEIAA GPI on cell cycling is attributed to the multifunctional mechanisms of electrorepulsion, physical hindrance from swollen PEIAA films to limit the vanadium crossover, and coordination chemistry of the chemically stable polymer interface with good wettability in strong acidic condition to maintain the concentrations of the vanadium active species stable in their respective half-cell reservoirs.

\section{Experimental Section}

4.1. Materials. Vanadium (IV) oxide sulfate $\left(\mathrm{VOSO}_{4} \bigotimes \mathrm{x}_{2} \mathrm{O}\right.$, 99.5\%, pure crystal) was purchased from Noah Technologies. The membrane $\left(\right.$ Nafion $\left.^{\mathrm{TM}} 115\right)$ was purchased from DuPont and pretreated in deionized (DI) water before use. The GCF (4 mm thick), and gasket (EPDM rubber sheet, $1 / 32$ in. thick) were purchased from the FuelCellStore. The PEI (branched, average $\mathrm{Mw} \sim 25,000$ ) and glutaraldehyde (GA) $\left(50 \%\right.$ in $\left.\mathrm{H}_{2} \mathrm{O}\right)$ were purchased at Sigma-Aldrich.

4.2. PEIAA Modification on GCF Electrodes. The crosslinked PEIAA was introduced to the surface of the GCF by sequential immersion in two different DI waters containing hyperbranched PEI $(25,000 \mathrm{Mw})$ and GA (50\% in solution), respectively. To get carboxylic acid groups in the PEI backbone, we used GA (1 wt\%). In this step, only one-fifth of a pristine GCF electrode $\left(5 * 6 \mathrm{~cm}^{2}, 4 \mathrm{~mm}\right.$ thickness $)$ was immersed in each solution one-by-one two times. The samples were stored at $70^{\circ} \mathrm{C}$ for 12 hours in an oven. During this time, the samples dried and the aldehyde groups oxidized to carboxylic acid groups on the polymer backbone. Then, we washed the samples in DI water several times to remove 
residual molecules. The dried PEIAA-GCF was cut into two pieces $\left(2 * 5 \mathrm{~cm}^{2}\right)$ for use as electrodes. Scheme S1 shows the schematic illustration of bare GCF and PEIAA-GCF samples.

4.3. Swelling Behavior and Zeta Potential Measurement. The PEI polymeric solution was mixed with GA solution in a weight ratio of 10:1. After it was washed and dried using the same procedures used for the PEIAA modification on GCF, a PEIAA sample was prepared to observe its swelling behavior in acidic conditions. The PEIAA sample was fully immersed in $0.9 \mathrm{M} \mathrm{H}_{2} \mathrm{SO}_{4}$ solution for 5 hours. To investigate the electrostatic force of PEIAA in acidic condition, a zeta potential measurement was conducted by Malvern Zetasizer Nano ZS with the Smoluchowski model. While the diluted PEI solution ( $0.5 \mathrm{wt} \%)$ with $200 \mathrm{~mL}$ of DI water was stirred at $800 \mathrm{rpm}$ in a round bottom flask using a magnetic spin bar, the diluted GA solution (1 wt\%) with $100 \mathrm{~mL}$ of DI water was slowly added over a 2-hour period using a dropping funnel. To remove the entangled particles, we used centrifugation at $3000 \mathrm{rpm}$. After drying and washing using the same procedure described previously, the PEIAA particles were dispersed in DI water and $0.9 \mathrm{M} \mathrm{H}_{2} \mathrm{SO}_{4}$ solution by ultrasonication for 1 hour. The colloidal solutions were directly used for the zeta potential measurement.

4.4. Electrochemical Measurement. The cyclic voltammetry $(\mathrm{CV})$ was carried out in $1.5 \mathrm{M} \mathrm{VOSO}_{4}$ in $3.5 \mathrm{M} \mathrm{H}_{2} \mathrm{SO}_{4}$ catholyte from 0.4 to $1.7 \mathrm{~V}$ at $25^{\circ} \mathrm{C}$. The pristine $\mathrm{GCF}$ and PEIAA-GCF samples were used as working electrodes attached with graphite electrode. The effective area of CF electrodes exposed to the catholyte is $6 \mathrm{~mm} \times 6 \mathrm{~mm}$. The glassy carbon and $\mathrm{Ag} / \mathrm{AgCl}$ were used as the counter and reference electrodes, respectively. The three-electrode system was purged with $\mathrm{N}_{2}$ gas for 10 min before the CV test. Electrochemical impedance spectroscopy (EIS) test was also operated over the frequency range of $0.1 \mathrm{~Hz}$ to $100 \mathrm{kHz}$ with an amplitude of $5 \mathrm{mV}$.

4.5. Ultraviolet-Visible (UV-vis) Spectroscopy Analysis. The concentration of $\mathrm{V}^{4+}$ in the $3.5 \mathrm{M} \mathrm{H}_{2} \mathrm{SO}_{4}$ solutions was analyzed using a UV-vis spectrophotometer (UXL-360, HI2608) by monitoring the maximum absorbance wavelength located at $765 \mathrm{~nm}$ (Figure S9) [32]. First, we performed a standard calibration analysis based on a series of known concentrations of $\mathrm{V}(\mathrm{IV}) \mathrm{OSO}_{4}$ solutions ranging from $0.01 \mathrm{M}$ to $0.2 \mathrm{M}$. Then, the concentration of $\mathrm{V}^{4+}$ samples was determined by comparison with the standard calibration curve (Figure S9a).

4.6. Redox Flow Battery Test. Electrochemical performances of PEIAA-GCF and pristine GCF electrodes were tested in a homemade flow cell with a $10 \mathrm{~cm}^{2}$ active area, which is controlled by an Arbin BT-2000 instrument for galvanic charge/discharge cycles within a $0.8 \mathrm{~V}-1.6 \mathrm{~V}$ voltage range at a constant current density of $50 \mathrm{~mA} \mathrm{~cm}^{-2}$. The flow rate of the electrolytes was $30 \mathrm{~mL} \mathrm{~min}^{-1}$ (the pump was a Cole Parmer, easy-load 2, 77202-60). Vanadium $3^{+}$and $4^{+}$ $\left(\mathrm{VO}^{2+}\right)$ solutions were prepared and used as the anolyte and catholyte (1.5 $\mathrm{M} \mathrm{VOSO}_{4}$ in $3.5 \mathrm{M} \mathrm{H}_{2} \mathrm{SO}_{4}$ ), respectively. N115 membranes were used for the cell test. Pristine GCF and PEIAA-GCF were used as the electrodes. Detailed descriptions of the cell design and testing procedures are described in previous publications [28, 33-35].

\section{Data Availability}

The data and materials used to support the findings of this study are available from the corresponding author upon request.

\section{Conflicts of Interest}

The authors declare no conflict of interest regarding the publication of this article.

\section{Authors' Contributions}

The work was supervised by W. Wang. H.-S. Lim and W. Wang conceived the idea. H.-S. Lim designed detailed experimental procedures, performed the material preparation, redox flow battery test, and data analysis, and wrote the paper. S. Chae made contribution on the BET study and drawing schematic illustration. L. Yan provided technical support for battery test. G. Li made contributions on the SEM and EDX studies. R. Feng performed CV and EIS. Y. Shin performed and analyzed FTIR of GCF electrodes. Z. $\mathrm{Nie}$ and Y. Liang provided technical support for electrolyte preparation. V. Murugesan managed XPS and ${ }^{1} \mathrm{H}$ NMR studies. B. Modachur Sivakumar and V. Shutthanandan performed and analyzed XPS. J. D. Bazak performed and analyzed ${ }^{1} \mathrm{H}$ NMR. X. Zhang performed and analyzed UV-vis. S. Kim made contribution on the diffusion test. All data are available in the main text or the supplementary materials.

\section{Acknowledgments}

The authors would like to acknowledge financial support primarily from the U.S. Department of Energy's Office of Electricity OE (under Contract No. 70247). The XPS and NMR and UV-vis measurements were performed at the Environmental Molecular Sciences Laboratory EMSL, a national scientific user facility sponsored by the DOE's Office of Biological and Environmental Research and located at Pacific Northwest National Laboratory (PNNL). PNNL is a multiprogram national laboratory operated by Battelle for DOE under Contract DE-AC05-76RL01830.

\section{Supplementary Materials}

Scheme S1 Schematic illustration of the bare GCF and PEIAA-GCF electrodes. Figure S1: dissolution test of PEI and PEIAA films in DI water at $70^{\circ} \mathrm{C}$. Figure S2: SEM images of (a) pristine GCF and (b) PEIAA-GCF samples. SEM-EDX (c) spectrum and element maps ((d) carbon, (e) oxygen, and (f) nitrogen) of a part of pristine GCF sample. Figure S3: thermogravimetric analysis curves of (a) pristine GCF and (b) PEIAA-GCF samples, performed in nitrogen gas. Figure S4: BJH adsorption pore size distribution based on the adsorption isotherm of (a) pristine GCF and (b) PEIAA- 
GCF samples. Figure S5: digital photographs of PEIAA chunk sample before (left) and after (right) immersing in $0.9 \mathrm{M} \mathrm{H}_{2} \mathrm{SO}_{4}$ solution for $5 \mathrm{~h}$. Figure $\mathrm{S} 6$ : digital photographs of (a) PEIAA particles and (b) PEIAA colloidal solutions prepared with DI water and $0.9 \mathrm{M} \mathrm{H}_{2} \mathrm{SO}_{4}$, respectively, and their (c) zeta potentials. Figure S7: (a) the three-electrode system for cyclic voltammetry (CV) test. (b) CV curves of pristine GCF and PEIAA-GCF electrodes under different scan rates $\left(0.5\right.$ and $\left.2.5 \mathrm{mV} \mathrm{s}^{-1}\right)$ in $1.5 \mathrm{M} \mathrm{VOSO}_{4}$ in $3.5 \mathrm{M}$ $\mathrm{H}_{2} \mathrm{SO}_{4}$. Figure S8: EIS spectra of vanadium redox flow cells with a pristine (a) GCF and (b) PEIAA-GCF electrodes and equivalent circuit. (c) Fitted parameters measured from the equivalent circuit models. Figure S9: (a) UV-vis spectra of the $\mathrm{V}(\mathrm{IV}) \mathrm{OSO}_{4}$ solutions ranging from $0.01 \mathrm{M}$ to $0.2 \mathrm{M}$ with a standard calibration curve and (b) UV-vis spectra of the $3.5 \mathrm{M}$ sulfuric acid solutions with (black) pristine GCF or (red) PEIAA-GCF electrode after 5 days with a flow rate of $30 \mathrm{~mL} \mathrm{~min}^{-1}$ for crossover test. Figure S10: SEM images of (a) fresh PEIAA-GCF positive electrode and (b) after 100 cycles carried out at a current density of $50 \mathrm{~mA} \mathrm{~cm}^{-2}$. Figure S11: (a) SEM image and SEM-EDX maps ((b) carbon, (c) oxygen, and (d) vanadium (e) nitrogen) of a part of PEIAA-GCF positive electrode after 100 cycles carried out at a current density of $50 \mathrm{~mA} \mathrm{~cm}^{-2}$. Figure S12: wide scan XPS spectra of pristine GCF electrodes (a) before and (b) after 100 cycles under constant current charge at $50 \mathrm{~mA} \mathrm{~cm}^{-2}$. Figure S13: SEM images of (a) fresh pristine GCF positive electrode and (b) after 100 cycles carried out at a current density of $50 \mathrm{~mA} \mathrm{~cm}^{-2}$. Figure S14: comparison of ${ }^{1} \mathrm{H}$ NMR spectra of catholytes in (blue and green) pristine GCF and (orange and red) PEIAA-GCF cells before and after 100 cycles carried out at a current density of $50 \mathrm{~mA} \mathrm{~cm}^{-2}$. Table S1: porous and surface properties of pristine GCF and PEIAA-GCF samples. (Supplementary Materials)

\section{References}

[1] W. Wang, Q. Luo, B. Li, X. Wei, L. Li, and Z. Yang, "Recent progress in redox flow battery research and development," Advanced Functional Materials, vol. 23, no. 8, pp. 970-986, 2013.

[2] P. Leung, X. Li, C. Ponce de León, L. Berlouis, C. T. J. Low, and F. C. Walsh, "Progress in redox flow batteries, remaining challenges and their applications in energy storage," RSC Advances, vol. 2, no. 27, pp. 10125-10156, 2012.

[3] M. Skyllas-Kazacos, M. H. Chakrabarti, S. A. Hajimolana, F. S. Mjalli, and M. Saleem, "Progress in flow battery research and development," Journal of the Electrochemical Society, vol. 158, no. 8, p. R55, 2011.

[4] S. Kim, J. Yan, B. Schwenzer et al., "Cycling performance and efficiency of sulfonated poly(sulfone) membranes in vanadium redox flow batteries," Electrochemistry Communications, vol. 12, no. 11, pp. 1650-1653, 2010.

[5] C. Jia, J. Liu, and C. Yan, “A significantly improved membrane for vanadium redox flow battery," Journal of Power Sources, vol. 195, no. 13, pp. 4380-4383, 2010.

[6] T. Sukkar and M. Skyllas-Kazacos, "Membrane stability studies for vanadium redox cell applications," Journal of Applied Electrochemistry, vol. 34, no. 2, pp. 137-145, 2004.
[7] D. G. Kwabi, Y. Ji, and M. J. Aziz, "Electrolyte lifetime in aqueous organic redox flow batteries: a critical review," Chemical Reviews, vol. 120, no. 14, pp. 6467-6489, 2020.

[8] X. Li, H. Zhang, Z. Mai, H. Zhang, and I. Vankelecom, "Ion exchange membranes for vanadium redox flow battery (VRB) applications," Energy \& Environmental Science, vol. 4, no. 4, pp. 1147-1160, 2011.

[9] R. A. Potash, J. R. McKone, S. Conte, and H. D. Abruña, "On the benefits of a symmetric redox flow battery," Journal of the Electrochemical Society, vol. 163, no. 3, pp. A338-A344, 2016.

[10] Q. Luo, L. Li, W. Wang et al., "Capacity decay and remediation of Nafion-based all-vanadium redox flow batteries," ChemSusChem, vol. 6, no. 2, pp. 268-274, 2013.

[11] L. Yu, D. Mu, L. Liu, and J. Xi, "Bifunctional effects of halloysite nanotubes in vanadium flow battery membrane," Journal of Membrane Science, vol. 564, pp. 237-246, 2018.

[12] A. B. Shah, X. Zhou, P. Brezovec, D. Markiewicz, and Y. L. Joo, "Conductive membrane coatings for high-rate vanadium redox flow batteries," ACS Omega, vol. 3, no. 2, pp. 1856$1863,2018$.

[13] J. Xi, W. Dai, and L. Yu, "Polydopamine coated SPEEK membrane for a vanadium redox flow battery," RSC Advances, vol. 5, no. 42, pp. 33400-33406, 2015.

[14] S. M. Ahn, H. Y. Jeong, J.-K. Jang et al., "Polybenzimidazole/ Nafion hybrid membrane with improved chemical stability for vanadium redox flow battery application," RSC Advances, vol. 8, no. 45, pp. 25304-25312, 2018.

[15] S. B. Lee, K. Mitra, H. D. Pratt et al., "Open data, models, and codes for vanadium redox batch cell systems: a systems approach using zero-dimensional models," Journal of Electrochemical Energy Conversion and Storage, vol. 17, no. 1, 2020.

[16] H. Zhang, X. Yan, L. Gao et al., "Novel triple tertiary amine polymer-based hydrogen bond network inducing highly efficient proton-conducting channels of amphoteric membranes for high-performance vanadium redox flow battery," ACS Applied Materials \& Interfaces, vol. 11, no. 5, pp. 5003-5014, 2019.

[17] D. Xing, S. Zhang, C. Yin, B. Zhang, and X. Jian, "Effect of amination agent on the properties of quaternized poly(phthalazinone ether sulfone) anion exchange membrane for vanadium redox flow battery application," Journal of Membrane Science, vol. 354, no. 1-2, pp. 68-73, 2010.

[18] Y. Zhao, M. Li, Z. Yuan, X. Li, H. Zhang, and I. F. J. Vankelecom, "Advanced charged sponge-like membrane with ultrahigh stability and selectivity for vanadium flow batteries," Advanced Functional Materials, vol. 26, no. 2, pp. 210-218, 2016.

[19] Q. Luo, H. Zhang, J. Chen, P. Qian, and Y. Zhai, “Modification of Nafion membrane using interfacial polymerization for vanadium redox flow battery applications," Journal of Membrane Science, vol. 311, no. 1-2, pp. 98-103, 2008.

[20] Y. Shi, C. Eze, B. Xiong et al., "Recent development of membrane for vanadium redox flow battery applications: a review," Applied Energy, vol. 238, pp. 202-224, 2019.

[21] T. N. L. Doan, T. K. A. Hoang, and P. Chen, "Recent development of polymer membranes as separators for all-vanadium redox flow batteries," RSC Advances, vol. 5, no. 89, pp. 72805-72815, 2015.

[22] X.-Z. Yuan, C. Song, A. Platt et al., "A review of all-vanadium redox flow battery durability: degradation mechanisms and 
mitigation strategies," International Journal of Energy Research, vol. 43, no. 13, pp. 6599-6638, 2019.

[23] A. M. Abu-Dief and I. M. A. Mohamed, "A review on versatile applications of transition metal complexes incorporating Schiff bases," Beni-Suef University Journal of Basic and Applied Sciences, vol. 4, no. 2, pp. 119-133, 2015.

[24] M. Marques, P. R. Pinto, C. T. de Andrade, and R. C. Michel, "Synthesis and characterization of low-molecular-weight polyacrolein," Journal of Applied Polymer Science, vol. 112, no. 3, pp. 1771-1779, 2009.

[25] C. Negrell, C. Voirin, B. Boutevin, V. Ladmiral, and S. Caillol, "From monomer synthesis to polymers with pendant aldehyde groups," European Polymer Journal, vol. 109, pp. 544-563, 2018.

[26] S. Hazra, M. Deb, and A. J. Elias, "Iodine catalyzed oxidation of alcohols and aldehydes to carboxylic acids in water: a metal-free route to the synthesis of furandicarboxylic acid and terephthalic acid," Green Chemistry, vol. 19, no. 23, pp. 5548-5552, 2017.

[27] S. Roy, X. Tang, T. Das et al., "Enhanced molecular level dispersion and interface bonding at low loading of modified graphene oxide to fabricate super nylon 12 composites," ACS Applied Materials \& Interfaces, vol. 7, no. 5, pp. 3142-3151, 2015.

[28] B. Li, Q. Luo, X. Wei et al., "Capacity decay mechanism of microporous separator-based all-vanadium redox flow batteries and its recovery," ChemSusChem, vol. 7, no. 2, pp. 577$584,2014$.

[29] K. H. Thompson and C. Orvig, "Coordination chemistry of vanadium in metallopharmaceutical candidate compounds," Coordination Chemistry Reviews, vol. 219-221, pp. 10331053, 2001.

[30] L. Deng, W.-T. Jin, W.-Z. Weng, and Z.-H. Zhou, "Interactions of vanadium with amino acids-monodentate coordination of vanadyl proline, lysine and histidine and catalytic degradations of methyl orange," Polyhedron, vol. 159, pp. 375-381, 2019.

[31] S. Ravi, S. Zhang, Y.-R. Lee et al., "EDTA-functionalized KCC1 and KIT-6 mesoporous silicas for $\mathrm{Nd}^{3+}$ ion recovery from aqueous solutions," Journal of Industrial and Engineering Chemistry, vol. 67, pp. 210-218, 2018.

[32] N. H. Choi, S. Kwon, and H. Kim, "Analysis of the oxidation of the V(II) by dissolved oxygen using UV-visible spectrophotometry in a vanadium redox flow battery," Journal of the Electrochemical Society, vol. 160, no. 6, pp. A973-A979, 2013.

[33] L. Estevez, D. Reed, Z. Nie et al., “Tunable oxygen functional groups as electrocatalysts on graphite felt surfaces for allvanadium flow batteries," ChemSusChem, vol. 9, no. 12, pp. 1455-1461, 2016.

[34] M. Vijayakumar, Q. Luo, R. Lloyd et al., "Tuning the perfluorosulfonic acid membrane morphology for vanadium redoxflow batteries," ACS Applied Materials \& Interfaces, vol. 8, no. 50, pp. 34327-34334, 2016.

[35] L. Li, S. Kim, W. Wang et al., "A stable vanadium redox-flow battery with high energy density for large-scale energy storage," Advanced Energy Materials, vol. 1, no. 3, pp. 394-400, 2011. 